\title{
Localized electron wave packet description of chemical bond and excitation: Floating and breathing Gaussian with valence-bond coupling
}

\author{
$\operatorname{AUTHOR}(S)$ :
}

Ando, Koji

\section{CITATION:}

Ando, Koji. Localized electron wave packet description of chemical bond and excitation: Floating and breathing Gaussian with valence-bond coupling. Computational and Theoretical Chemistry 2017, 1116: 159-162

ISSUE DATE:

2017-09-15

URL:

http://hdl.handle.net/2433/226741

\section{RIGHT:}

(C) 2017. This manuscript version is made available under the CC-BY-NC-ND 4.0 license

http://creativecommons.org/licenses/by-nc-nd/4.0/; The full-text file will be made open to the public on 15 September 2019 in accordance with publisher's 'Terms and Conditions for Self-Archiving'; この論文は出版社版でありません。引用 の際には出版社版をご確認ご利用ください。; This is not the published version. Please cite only the published version. 


\title{
Localized electron wave packet description of chemical bond and excitation: Floating and breathing Gaussian with valence-bond coupling
}

\author{
Koji Ando* \\ Department of Chemistry, Graduate School of Sciences, Kyoto University, Sakyo-ku, Kyoto 606-8502, Japan
}

\begin{abstract}
A model of localized electron wave packets (WPs) with variable position and width (floating and breathing) that are spin-coupled as per the valence-bond theory is presented. It produces accurate potential energy curves of $\mathrm{LiH}$ in the ground singlet and triplet states. Quantization in a mean-field approximation of the motion of a WP that corresponds to the Li 2 s electron generates semi-quantitative potential energy curves of low energy excited states. Real-time semiquantal dynamics of the WP induced by an intense laser pulse gives high-harmonic generation spectra that capture qualitative features of a higher-level wave function calculation.
\end{abstract}

\section{INTRODUCTION}

The valence-bond (VB) theory provides many useful concepts for understanding formation and reactivity of chemical bond [1-8]. The standard VB method is based on the linear combination of atomic orbitals (LCAO) where the AOs are clumped at the nuclear centers and the orbital exponents are fixed at predetermined values. Variation of the electronic wave function is described by superposing a number of AOs of different orbital exponents and angular momenta.

The LCAO model is useful particularly for static problems but is not imperative. We have been studying in recent years a model of floating and breathing (variable position and width) Gaussian wave packets (WPs) with non-orthogonal VB coupling [9-11]. It was designed to be combined with nuclear WPs in a semiquantal molecular dynamics (MD) simulation [12-15]. In order to take account of the anti-symmetry of many-electron wave functions retaining the localized WP picture, the VB model seemed appropriate. The localized WP model was envisaged to have merits for studying real-time electron dynamics.

In our previous studies, the WP-VB model was found to give reasonably accurate potential energy surfaces of the electronic ground states of small molecules such as $\mathrm{H}_{2}, \mathrm{LiH}$, $\mathrm{BeH}_{2}, \mathrm{H}_{2} \mathrm{O}$, and $\mathrm{NH}_{3}$ with the minimal numbers of electron WPs [9-11]. The accuracy came from the flexibility to describe the static correlation by the VB coupling, the dynamic correlation by the WP breathing, and the polarization by the WP floating. The electronic excited states have been also examined by quantizing the motion of WP center [11] in an idea similar to the propagator theory [16]. Furthermore, the nuclear and electronic WPs were combined (with static electrons in the Born-Oppenheimer approximation) to the semiquantal MD simulation of liquid hydrogen, which gave quantitative diffusion constant and viscosity at temperatures below $25 \mathrm{~K}$ [12,-15].

This work extends the study on LiH in Ref. [11] to examine the potential energy surfaces for the electron WP motion and the real-time dynamics induced by an intense laser pulse. The

\footnotetext{
*E-mail: ando@kuchem.kyoto-u.ac.jp
}

study of real-time electron dynamics is an emerging field of recent research with the advent of attosecond time-resolved laser spectroscopy [17, 18].

In Sec. 2, the theory is outlined. In Sec. 3.1, the potential energy curves for the electron WP motion are examined. In Sec. 3.2, the laser-induced electron dynamics and the emission of high-harmonic generation spectra are studied. Section 4 concludes.

\section{THEORY}

As in the standard VB methods, the electronic wave function is assumed to be an antisymmetrized product of spatial and spin functions,

$$
\Psi(1, \cdots, N)=\mathcal{A}\left[\Phi\left(\boldsymbol{r}_{1}, \cdots, \boldsymbol{r}_{N}\right) \Theta(1, \cdots, N)\right],
$$

with the spatial part modeled by a product of one-electron functions,

$$
\Phi\left(\boldsymbol{r}_{1}, \cdots, \boldsymbol{r}_{N}\right)=\phi_{1}\left(\boldsymbol{r}_{1}\right) \cdots \phi_{N}\left(\boldsymbol{r}_{N}\right) .
$$

In contrast with the conventional VB methods that employ atomic orbitals clumped at the nuclear centers, we apply floating and breathing spherical Gaussian WPs of a form [19, 20],

$$
\phi(\boldsymbol{r}, t)=\left(2 \pi \rho_{t}^{2}\right)^{-\frac{3}{4}} \exp \left[-\gamma_{t}\left|\boldsymbol{r}-\boldsymbol{q}_{t}\right|^{2}+i \boldsymbol{p}_{t} \cdot\left(\boldsymbol{r}-\boldsymbol{q}_{t}\right) / \hbar\right],
$$

in which the complex coefficient

$$
\gamma_{t}=\frac{1}{4 \rho_{t}^{2}}-\frac{i}{2 \hbar} \frac{\pi_{t}}{\rho_{t}}
$$

contains the time-dependent WP width $\rho_{t}$ and its conjugate momentum $\pi_{t}$ (see Eq. (7p below). $\boldsymbol{q}_{t}$ and $\boldsymbol{p}_{t}$ denote the position of the WP center and its conjugate momentum. The spin part $\Theta(1, \cdots, N)$ consists of the spin eigenfunctions. In this work, we employ a single configuration of the perfect-pairing form,

$$
\Theta=\theta(1,2) \cdots \theta\left(N_{p}-1, N_{p}\right) \alpha\left(N_{p}+1\right) \cdots \alpha(N),
$$

with $\theta(i, j)=(\alpha(i) \beta(j)-\beta(i) \alpha(j)) / \sqrt{2}$ for paired $N_{p}$ electrons. 
The ground state wave function and energy in a given spin multiplicity is computed by optimizing the WP center and width, $\boldsymbol{q}$ and $\rho$, to minimize the energy expectation, $E=$ $\langle\Psi|\hat{H}| \Psi\rangle /\langle\Psi \mid \Psi\rangle$, with the momentum variables $\boldsymbol{p}$ and $\pi$ nullified [21]. For the excited states, the standard method would be the configuration-interaction (CI) of multiple VB configurations [16]. Alternatively, we are working on a propagator theory with the coherent-state path-integral representation [22], exploiting the fact that the Gaussian WP is a representation of the (over)complete coherent-state [21, 23, 24]. In Sec. 3 , a simplified calculation based on this idea with the singleparticle mean-field approximation is presented.

In the single particle approximation, the equations of motion for the WP center and width have a simple Hamiltonian form [19, 20, 25, 26],

$$
\begin{array}{ll}
\dot{q}=\frac{\partial\langle\hat{H}\rangle}{\partial p}, & \dot{p}=-\frac{\partial\langle\hat{H}\rangle}{\partial q}, \\
\dot{\rho}=\frac{\partial\langle\hat{H}\rangle}{\partial \pi}, & \dot{\pi}=-\frac{\partial\langle\hat{H}\rangle}{\partial \rho},
\end{array}
$$

with the Hamiltonian function

$$
\langle\hat{H}\rangle=\frac{p^{2}}{2 m}+\frac{\pi^{2}}{2 m}+\frac{\hbar^{2}}{8 m \rho^{2}}+\langle V\rangle,
$$

where $m$ is the mass of electron and $\langle\cdots\rangle$ is the expectation value with the WP $\phi(\boldsymbol{r}, t)$. They are derived from the time-dependent variational principle. For many-electron wave functions with non-orthogonal WPs, the equations become much more complicated, which we shall leave out of the scope of this paper.

\section{NUMERICAL CALCULATION AND DISCUSSION}

\section{Bond Formation and Excitation}

Figure 1 displays the computed potential energy curves of the ground and excited states of $\mathrm{LiH}$ in spin singlet and triplet. The excited states were calculated by quantizing the motion of the center of a WP that corresponds to the Li 2 s electron: we first constructed potential energy surfaces along displacements of the WP centers (Fig. 2 to be discussed later) and then numerically solved the time-independent Schrödinger equation in a single-particle mean-field approximation. For comparison, results from the multi-reference $\mathrm{CI}$ with single and double excitations (MRCISD) [27] are included in the figure. The agreement is quantitative for the singlet and triplet ground states, $X^{1} \Sigma^{+}$and $a^{3} \Sigma^{+}$. For the excited states, the correspondence appears semi-quantitative. More elaborate calculations that will be noted in the concluding section will improve the agreement. Nevertheless, we proceed with the current framework as it offers a simple intuitive picture for the electron excitation dynamics.

Figure 2 displays the potential energy curves for the WP motion along the bond direction in the ground singlet (a) and

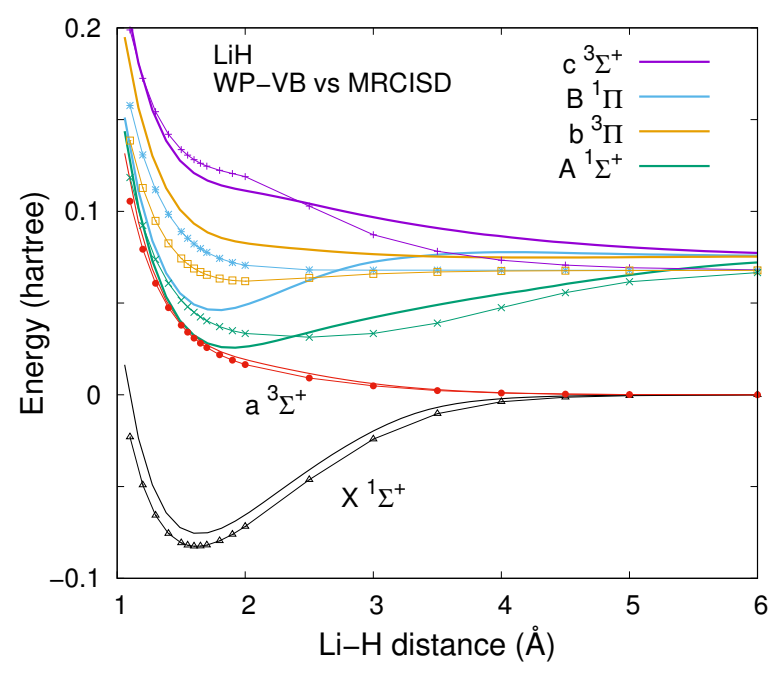

FIG. 1: Potential energy curves of the ground and excited singlet and triplet states of $\mathrm{LiH}, X^{1} \Sigma^{+}, A^{1} \Sigma^{+}, B^{1} \Pi, a^{3} \Sigma^{+}, b^{3} \Pi$, and $c^{3} \Sigma^{+}$. The solid lines are from the WP-VB calculation and the symbols are from the MRCISD calculation with the cc-pVDZ basis set.

triplet (b) states. At the origin is the Li nucleus, and the proton is at $1.6 \AA$. In this calculation, the center of each WP was displaced while the other WPs are fixed, which means that the potential energy of each WP was evaluated under the meanfield of others. Figures 2(a) and (b) both indicate that the two WPs that correspond to the Li 1s electrons are deeply bound and contracted at the Li nucleus. The WP that corresponds to the $\mathrm{H} 1 \mathrm{~s}$ electron is bound to the proton with the potential well shallower than those for the Li 1s electrons. The WP that corresponds to the $\mathrm{Li} 2 \mathrm{~s}$ electron is near the $\mathrm{H}$ nucleus in the singlet state, whereas in the triplet state it is behind the $\mathrm{Li}$ nucleus seen from the $\mathrm{H}$ atom. The inset displays the WPs by the circles of the radius $\rho$. It has been demonstrated in Fig. 2 of Ref. [11] that these WP configurations correspond well with the standard molecular orbitals. The potential well for the Li 2s WP is the shallowest in both singlet and triplet states. The difference of the curvature from the others supports the single-electron treatment for the calculation of the low energy excitations in Fig. 1. Hereafter, we loosely denote 'Li 2s WP' etc. rather than 'WP that corresponds to the $\mathrm{Li} 2 \mathrm{~s}$ electron', although the correspondence is only intuitive as the WPs are not clumped at the nuclear center and the size is variable.

The potential energy curves along the displacement perpendicular to the bond that determine the $\Pi$ excited states are simple symmetric single wells (and thus were omitted from the figure) of varying depth. Here again, the potential well for the Li 2 s electron is the shallowest with the smallest curvature that determines the lowest $\Pi$ states in Fig. 1.

We note that the WP widths were fixed at the values optimized for the ground state when constructing the potential energy curves in Fig. 2. This is also the case in the calculation of excitation energies at each $\mathrm{LiH}$ distance in Fig. 1. Alternatively, we might consider variation of the WP width for 

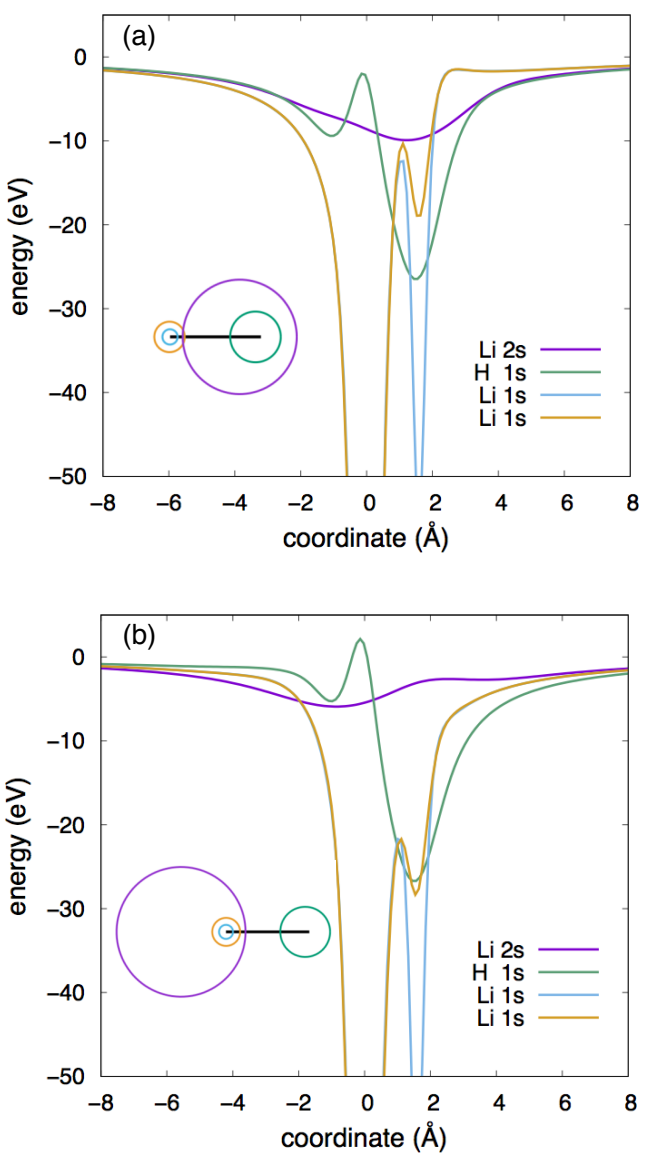

FIG. 2: Potential energy curves for the motion of wave packet centers in (a) the singlet $X^{1} \Sigma^{+}$state and (b) the triplet $a^{3} \Sigma^{+}$state. The insets are the wave packets represented by circles with the radius of the WP width $\rho$.

quantization. We might also optimize adiabatically the WP width along the variation of the WP center. Nonetheless, the physical meanings of these procedures have not been clarified. A related study on the WP width variation in the initial-value represented propagator has been reported [22].

\section{Electron Dynamics and High-Harmonic Generation}

As described in Sec. 2, the computation of single-particle dynamics is straightforward. Here we focus on the Li 2 s WP that is the most labile to the external field, and compute its response dynamics to a laser pulse of time-dependent electric field,

$$
E(t)=E_{0} \sin \left(\omega_{0} t\right) \sin ^{2}(\pi t / \tau) \quad(0 \leq t \leq \tau)
$$

The parameters were taken from Ref. [28] that employed the time-dependent complete-active-space CI (TD-CASCI); the frequency $\omega_{0}$ corresponds to the wavelength of $750 \mathrm{~nm}$ and the duration $\tau$ of three optical cycles, $\tau=3\left(2 \pi / \omega_{0}\right) \simeq 7.51$

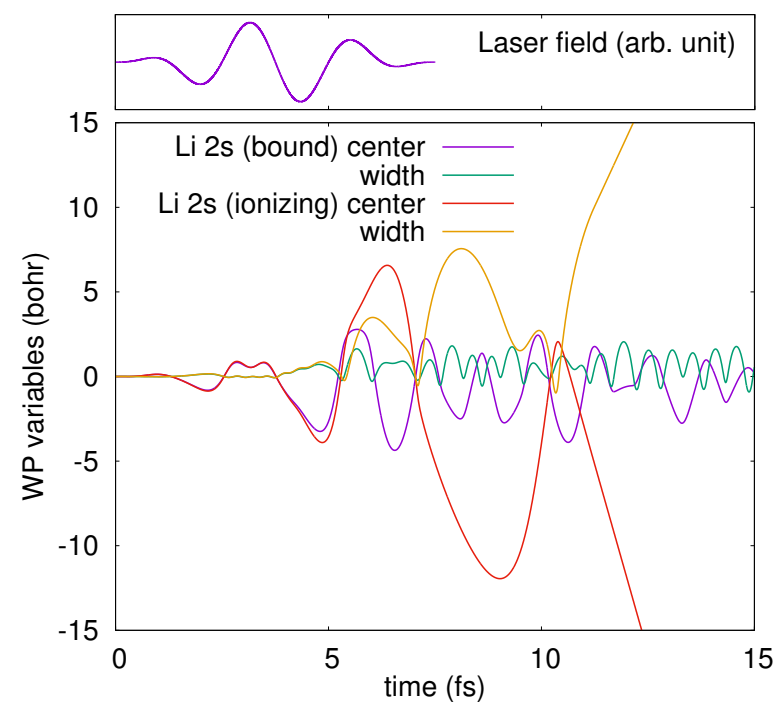

FIG. 3: Trajectories of the wave packet center and width induced by the pulse laser field displayed in the upper panel.

fs. We used weaker field intensity $E_{0}$ than in Ref. [28]; -1.54 and $-1.48 \mathrm{~V} / \mathrm{cm}$, that is, the laser intensity of $1.12 \times 10^{14}$ and $1.08 \times 10^{14} \mathrm{~W} / \mathrm{cm}^{2}$, as these values were found to be just above and below the threshold of ionizing trajectories for the present model. The positive direction of the field was defined to be that from $\mathrm{Li}$ to $\mathrm{H}$. Here, the $\mathrm{Li}-\mathrm{H}$ bond length was set to be 2.3 bohr following Ref. [28]. At $t=0$, the LiH molecule is in the singlet ground state. We added the Lorentz force from the electric field to the equations of motion (6) for the WP center.

Figure 3 displays the ionizing and non-ionizing (bound) trajectories of the WP center and width. In the initial time up to $\sim 5.7 \mathrm{fs}$, both the ionizing and bound trajectories of the WP center mostly follow the time profile of the laser field, except the small hump at $t=3.2 \mathrm{fs}$. Then, the ionizing trajectory starts to oscillate in an amplitude as large as $12 \mathrm{bohr}$, recombines to the molecule, and dissociates after $10 \mathrm{fs}$. Associated with this large amplitude oscillation of the WP center, the WP width broadens when the WP center departs from the molecule, and spreads to the free electron state. In the bound trajectory, the WP center remains around the molecule, but the oscillation amplitude of $\sim 5$ bohr is about twice the $\mathrm{Li}-\mathrm{H}$ bond length of 2.3 bohr.

Figure 4 displays the Fourier transformed dipole acceleration that gives the high-harmonic generation (HHG) spectrum [29, 30]. For comparison, the result from the TD-CASCI of Ref. [28] is included. The present results do not exhibit clear plateau and cut-off that are considered to be the characteristics of the laser-induced HHG spectra. These features have been discussed in terms of the ponderomotive energy of fieldinduced dynamics and the three-step model of ion recombination [31-33]. Their absence in the present semiquantal calculations could be due to the insufficient account of the quantum interference in the ion recombination dynamics. However, 


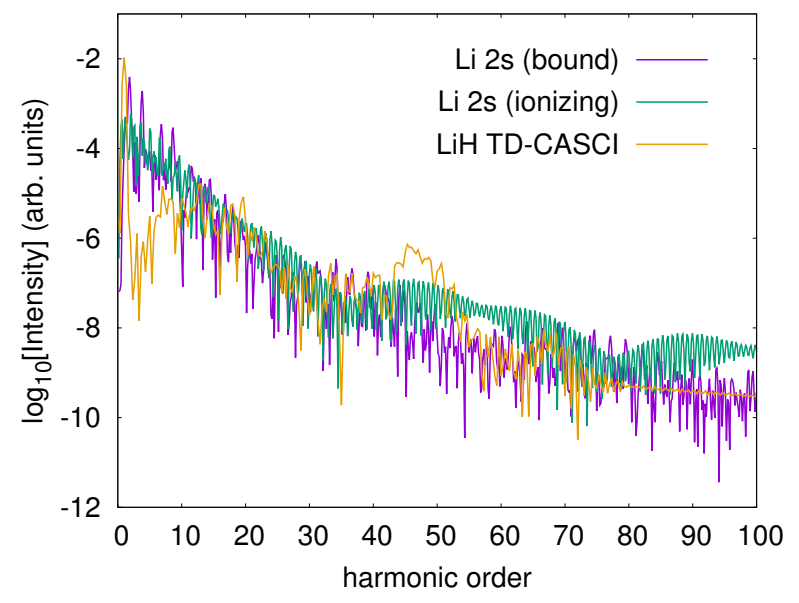

FIG. 4: Fourier transform of the dipole acceleration that gives the HHG spectra. The abscissa is the harmonic-order $\omega / \omega_{0}$. The TDCASCI data is from Ref. [28].

other qualitative features such as the alternating peak structure intense at the odd harmonic orders and the spectral intensity extending up to a hundred of harmonic order were reproduced.

\section{CONCLUSION}

A model of floating and breathing electron wave packets with the non-orthogonal valence-bond spin-coupling has been presented. Although it is still in need of further developments, its potential would have been clarified. We anticipate that the remaining tasks will be more technical than fundamental. For instance, more flexible forms of the localized WP than the spherical Gaussian should be examined. The first candidate would be the ellipsoidal Gaussian function: we have reported its use for nuclear WPs in the simulation of liquid water [34], but its application to many-electrons involves a numerical bottleneck in the evaluation of two-electron integrals. We also need to establish the propagator theory with the coherent-state representation and its implementation in order to improve the accuracy of quantum dynamics. Works on these are currently under way and will be reported in due course.

\section{ACKNOWLEDGMENT}

The author is grateful to Professors Takeshi Sato and Kenichi Ishikawa for providing their data in Fig. 4. This work was supported by KAKENHI No. 26248009 and 26620007.

[1] L. Pauling, The Nature of the Chemical Bond, Cornell University Press, New York, 1960.
[2] D. L. Cooper, J. Gerratt, M. Raimondi, The electronic structure of the benzene molecule, Nature 323 (1986) 699-701.

[3] A. Warshel, Computer Modeling of Chemical Reactions in Enzymes and Solution, Wiley, New York, 1991.

[4] D. G. Truhlar, Valence bond theory for chemical dynamics, J. Comp. Chem. 28 (2006) 73-86.

[5] S. S. Shaik, P. C. Hiberty, A Chemist's Guide to Valence Bond Theory, Wiley, New Jersey, 2008.

[6] W. Wu, P. Su, S. Shaik, P. C. Hiberty, Classical valence bond approach by modern methods, Chem. Rev. 111 (2011) 75577593.

[7] D. W. Small, M. Head-Gordon, Post-modern valence bond theory for strongly correlated electron spins, Phys. Chem. Chem. Phys. 13 (2011) 19285-19297.

[8] P. B. Karadakov, D. L. Cooper, B. J. Duke, J. Li, Spin-coupled theory for ' $n$ electrons in $m$ orbitals' active spaces, J. Phys. Chem. A 116 (2012) 7238-7244.

[9] K. Ando, Semiquantal valence-bond wavepacket description of chemical bonding, Bull. Chem. Soc. Jpn. 82 (2009) 975-983.

[10] K. Ando, Electron wave packet modeling of chemical bonding: Floating and breathing minimal packets with perfect-pairing valence-bond spin coupling, Chem. Phys. Lett. 523 (2012) 134138.

[11] K. Ando, A corpuscular picture of electrons in chemical bond, J. Chem. Phys. 144 (2016) 124109-1-4.

[12] K. Hyeon-Deuk, K. Ando, Quantum molecular dynamics simulation of liquid para-hydrogen by nuclear and electron wave packet approach, J. Chem. Phys. 140 (2014) 171101-1-4.

[13] K. Hyeon-Deuk, K. Ando, Correlations of intra- and intermolecular dynamics and structure in liquid para-hydrogen, Phys. Rev. B 90 (2014) 165132-1-5.

[14] K. Hyeon-Deuk, K. Ando, Dynamical and structural analyses of solid hydrogen under vapor pressure, J. Chem. Phys. 144 (2015) 171102-1-4.

[15] K. Hyeon-Deuk, K. Ando, Distinct structural and dynamical difference between supercooled and normal liquids of hydrogen molecules, Phys. Chem. Chem. Phys. 18 (2016) 2314-2318.

[16] R. McWeeny, Methods of Molecular Quantum Mechanics, Academic, London, 1992.

[17] M. F. Kling, M. J. J. Vrakking, Attosecond electron dynamics, Ann. Rev. Phys. Chem. 59 (2008) 463-492.

[18] F. Krausz, M. Ivanov, Attosecond physics, Rev. Mod. Phys. 81 (2009) 163-234.

[19] F. Arickx, J. Broeckhove, E. Kesteloot, L. Lathouwers, P. van Leuven, Dynamics of wave packets and the time-dependent variational principle, Chem. Phys. Lett. 128 (1986) 310-314.

[20] A. K. Pattanayak, W. C. Schieve, Gaussian wave-packet dynamics: Semiquantal and semiclassical phase-space formalism, Phys. Rev. E 50 (1994) 3601-3614.

[21] Y. Tsue, Maslov phase as geometric phase in the timedependent variational approach with squeezed coherent states, Prog. Theor. Phys. 88 (1992) 911-932.

[22] K. Ando, Initial value represented propagator for semiquantal squeezed state wave packet, Chem. Phys. Lett. 591 (2014) 179184.

[23] H. Kuratsuji, A path integral formalism of collective motion, Prog. Theor. Phys. 65 (1981) 224-240.

[24] J. R. Klauder, B. S. Skagerstam (Eds.), Coherent States: Applications in Physics and Mathematical Physics, World Scientific, Singapore, 1985.

[25] K. Ando, Semiquantal time-dependent hartree approach to condensed phase chemical dynamics: Application to system-bath model, J. Chem. Phys. 121 (2004) 7136-7143.

[26] O. V. Prezhdo, Quantized hamilton dynamics, Theor. Chem. 
Acc. 116 (2006) 206-218.

[27] We used the program GAMESS: M. W. Schmidt, K. K. Baldridge, J. A. Boatz, S. T. Elbert, M. S. Gordon, J. H. Jensen, S. Koseki, N. Matsunaga, K. A. Nguyen, S. Su, T. L. Windus, M. Dupuis, J. A. Montgomery Jr, General atomic and molecular electronic structure system, J. Comput. Chem. 14 (11) (1993) 1347-1363.

[28] T. Sato, K. L. Ishikawa, Time-dependent multiconfiguration self-consistent-field method based on the occupation-restricted multiple-active-space model for multielectron dynamcis in intese laser fields, Phys. Rev. A 91 (2015) 023417-1-15.

[29] J. C. Baggesen, L. B. Madsen, On the dipole, velocity and acceleration forms in high-order harmonic generation from a single atom or molecule, J. Phys. B: At. Mol. Opt. Phys. 44 (2011) 115601-1-5.

[30] A. D. Bandrauk, F. Fillion-Gourdeau, E. Lorin, Atoms and molecules in intense laser fields: gauge invariance of theory and models, J. Phys. B: At. Mol. Opt. Phys. 46 (2013) 153001$1-33$.

[31] J. L. Krause, K. J. Schafer, K. C. Kulander, High-order harminic generation from atoms and ions in the high intensity regime, Phys. Rev. Lett. 68 (1992) 3535-3538.

[32] K. J. Schafer, B. Yang, L. F. DiMauro, K. C. Kulander, Above threshold ionization beyond the high harmonic cutoff, Phys. Rev. Lett. 70 (1993) 1599-1602.

[33] P. B. Corkum, Plasma perspective on strong-field multiphoton ionization, Phys. Rev. Lett. 71 (1993) 1994-1997.

[34] J. Ono, K. Ando, Semiquantal molecular dynamics simulation of hydrogen-bond dynamics in liquid water using multidimensional gaussian wave packets, J. Chem. Phys. 137 (2012) 174503-1-18. 